\title{
A Regrettable Instance of Medical Abortion How to Get-Going?
}

\author{
Josie Baral, Ashma Rana, Geeta Gurung \\ Dept Obs/Gyn TUTH
}

\section{Dear Editor}

The positive aspect our country has played in the improvement of women's health issues are admirable by adopting new method of contraception and also by taking keen interest to promote safe abortion practices and services.

However what is lagging behind is the way how the medical abortion (MA) medication is being sold and who sells them. And finally who takes the sole responsibility should a mishap occurs?

We are going to cite an example of a woman who was Para 5 and was admitted for severe vaginal bleeding from the emergency Unit of TUTH. History indicated that she had taken some pill for abortion induction from local pharmacy at Balaju. Her Hb was $3 \mathrm{gm} \%$ and was transfused with 4 pint of packed cell and completeness of uterine evacuation was accomplished. This is just one example. There are many others.

We must rethink whether we are in process for maternal morbidity and mortality reduction or preparing a new cause for the same. As all of us are aware, the role NESOG has played in the introduction of medical abortion (MA) in Nepal initially. In the year 2007-2008 NESOG with technical collaboration with Gynuity conducted a clinical trial (400 women) on MA up to 63 days in four centres achieving success rate of $91 \%$.
NESOG has been one of the founder member in MoHP formed Task Force to "develop a national strategy for implementation of medical abortion" Feb 2008 along with FPAN, MSI, Ipas. And also to the fact in addition that NESOG also worked in the development of training curricula under the banner of National Health Training Centre (NHTC), with the technical support from Ipas. Ipas data details of 24 ANMs trained as SBAs who received three days MA training from PHCCs and Health Posts in January 2010. Recently 128 ANM have been trained to cover 10 districts by IPAS, which can be counted as positive effort that would probably answer our concern that the drug is properly advocated. And as of date MA has been introduced in sites accessible for CEOC facility.

Proposed route for misoprostol are oral (swallowed) buccal/sublingual and vaginal in doses or $400-800 \mathrm{mcg}$ preceded $24-48 \mathrm{~h}$ by oral mifepristone at fixed doses of $200 \mu \mathrm{gm}$. This dosing pattern has to be endorsed to avoid complication.

Before this, also the Contraindication of medical abortion must be considered.

In conclusion, misoprostol, the miracle pill is suggested to be advocated only after proper counseling by trained personnel as giving the drug over the counter could endanger women's life at times.

\author{
Correspondence \\ Josie Baral PHD \\ Assoc Prof Dept of Obs/Gyn TU Teaching Hosp, IOM \\ Phone : 9851104335 \\ Email:baraljyoti5@hotmail.com
}

